\title{
Measuring the cosmological density perturbation
}

\author{
S. Sarkara * \\ ${ }^{a}$ Rudolf Peierls Centre for Theoretical Physics, \\ University of Oxford, Oxford OX1 3NP, UK
}

Precision measurements of anisotropies in the cosmic microwave background and of the clustering of largescale structure have supposedly confirmed that the primordial density perturbation has a (nearly) scale-invariant spectrum. However this conclusion is based on assumptions about the world model and the nature of the dark matter. Physical models of inflation suggest that the spectrum may not in fact be scale-free, which would imply rather different cosmological parameters on the basis of the same observational data.

\section{Introduction}

The primordial density perturbation of the universe is the earliest relic we have of the Big Bang, although it is not clear exactly when it was generated. It was certainly before the primordial nucleosynthesis era, when the expansion is known to have been radiation-dominated, and it must have been well below the Planck era, from the absence of a significant imprint of gravitational waves on the cosmic microwave background (CMB). Long before any relevant observations were available, it was argued from general considerations of the formation of large-scale structure (LSS) through gravitational instability that the density perturbation should have a scale-invariant 'Harrison-Zeldovich' (H-Z) form:

$P(k)=\left\langle\left|\delta_{k}\right|^{2}\right\rangle=A k^{n}, \quad$ with $n=1$,

where $\delta_{k} \equiv \int[\delta \rho(\vec{x}) / \bar{\rho}] \mathrm{e}^{-i \vec{k} \cdot \vec{x}} \mathrm{~d}^{3} x$ is the Fourier transform of spatial fluctuations in the density field of wavelength $\lambda=2 \pi / k$. It was also anticipated that this growth occurs in a sea of dark matter which dominates over baryonic matter, since otherwise structure can form only after the universe becomes neutral which is insufficient time, given the extant upper limits on the amplitude of the 'seed fluctuations' from the absence of large anisotropies in the CMB [1].

\footnotetext{
*Talk at Workshop on The Density Perturbation in the Universe, Demokritos Centre, Athens, 25-26 June 2004
}

As is well known, powerful support for both conjectures came from the theory of inflation which can both generate density perturbations on (apparently) super-horizon scales with an approximately scale-invariant spectrum, and also creates a spatially flat universe which would require there to be a large amount of dark matter, since the baryonic component is known to be small: $\Omega_{\mathrm{B}} h^{2} \simeq 0.012-0.025$ from considerations of primordial nucleosynthesis [2, where $h \equiv H_{0} / 100 \mathrm{Km} \mathrm{s}^{-1} \mathrm{Mpc}^{-1}$ is the Hubble paramter. The detection by COBE of large-angle anisotropies in the CMB generated by the SachsWolfe effect provided the normalization of the amplitude of the primordial perturbations and confirmed that structure does grow through gravitational instability and not e.g. through explosive events (which were constrained additionally by the stringent limits set by $\mathrm{COBE}$ on the associated spectral distortions of the $\mathrm{CMB}$ ). It then became clear that if the primordial density perturbation does have a H-Z form, then the amplitude of matter fluctuations on cluster and galaxy scales is too high relative to observations, if we inhabit a critical density, cold dark matter (CDM) dominated universe 3 .

As illustrated in Fig. 1 several solutions were proposed to address this problem. One could invoke a small admixture of hot dark matter (HDM) in the form of neutrinos with a mass of $\mathcal{O}(\mathrm{eV})$ to damp small-scale power - this was the "Mixed Dark Matter Model" (MDM). Al- 


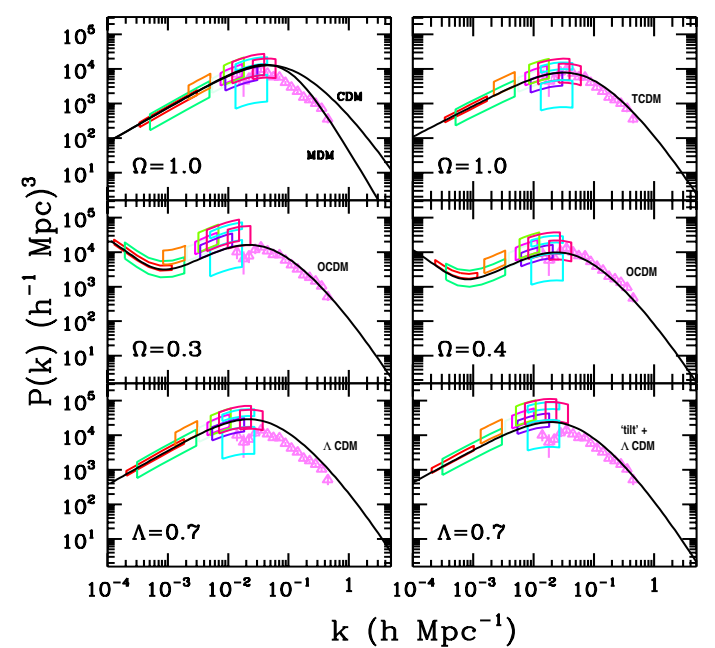

Figure 1. The matter power spectrum inferred from LSS and CMB data (circa 1995) compared with theoretical models 4. As seen top left, the excess small-scale power in the COBE-normalized standard CDM model $\left(n=1, \Omega_{\mathrm{B}}=0.03, h=\right.$ $0.5)$ is suppressed in the MDM model which has $\Omega_{\nu}=0.3 \mathrm{in} \sim 2 \mathrm{eV}$ mass neutrinos. Alternatively, this can be done by tilting the spectrum as in the TCDM model with $n=0.9, \Omega_{\mathrm{B}}=0.1$ and $h=$ 0.45 shown top right. The middle panels show open universe (OCDM) models and the bottom panels show $(\Lambda \mathrm{CDM})$ models of a flat universe with a cosmological constant.

ternatively one could appeal to dynamical measurements that suggested a matter content short of the critical value, $\Omega_{\mathrm{m}} \sim 0.3-$ in such an "Open Cold Dark Matter Model" (OCDM), the epoch of matter-domination occurs later so there is less time for structure to grow, thus suppressing power on the relevant scales. Of course if one believed that the universe is spatially flat as is generally expected from inflation then it was necessary to invoke a compensating cosmological constant with $\Omega_{\Lambda} \sim 0.7$. It is this "Lambda Cold Dark Matter Model" $(\Lambda \mathrm{CDM})$ that subsequently gained credence from observations of the Hubble diagram of SN Ia which suggested that the expansion is accelerating due to just such a cosmological constant [5]. More recently it has been promoted as the 'standard model' of the universe on the basis of accurate measurements of $\mathrm{CMB}$ anisotropies and of the properties of LSS [6]. However it is important to recall that the data can be fitted without altering the world model - the small-scale power is naturally suppressed if the primordial spectrum is 'tilted' below the scale-invariant form. This "Tilted Cold Dark Matter Model" (TCDM) shown in Fig. 1 found theoretical motivation in models of slowroll 'new inflation' based on $N=1$ supergravity which predicted just such a spectrum (with logarithmic $k$-dependent corrections) 78].

Subsequently it was widely advertised that precision measurements of CMB anisotropy can be used to determine cosmological parameters with unprecedented accuracy [9] but it was still the case that this required assumptions about the spectrum of the primordial density perturbation. An useful analogy is to see the generation of CMB anisotropy and the formation of LSS as a cosmic scattering experiment, in which the primordial density perturbation is the 'beam', the universe itself is the 'detector' and its matter content is the 'target'. In complete contrast to the situation in the laboratory, neither the properties of the beam, nor the parameters of the target or even the detector are known - only the actual 'interaction' is known to be gravity. Clearly the inverse problem of reconstructing the primordial density perturbation from the CMB and LSS data is necessarily uncertain due to our ignorance of the nature of the dark matter and of cosmological parameters.

\section{The present situation}

Nevertheless it was hoped that with sufficiently precise data and the inclusion of external constraints on cosmological parameters such as $h$, these 'degeneracies' could be resolved [10]. WMAP, the successor to COBE, has indeed provided a much more precise measurement of $\mathrm{CMB}$ anisotropy, down to sub-degree scales; the angular power spectrum is consistent, for an assumed scale-free power-law primordial spectrum, with 
a flat $\Lambda$ CDM model having $\Omega_{\mathrm{m}}=0.29 \pm 0.07$, $h=0.72 \pm 0.05, \Omega_{\mathrm{B}}=0.047 \pm 0.006$, and $n=0.99 \pm 0.0 .04$ [1]. Moreover the implied matter power spectrum matches the power spectrum of galaxy clustering from the $2 \mathrm{dF}$ redshift survey [12, indicating that any 'bias' between visible and dark matter is small. This world model is said to be concordant with parameters derived from the SN Ia Hubble diagram [13, with the measurement of $h$ by the Hubble Key Project [14, and with a variety of other cosmological probes (e.g. weak gravitational lensing, cluster baryon fraction, peculiar velocity fields, etc). It is indeed tempting to believe that we now have a 'standard model' for cosmology.

It is however important to keep in mind that a very different world model can also be made compatible with the CMB and LSS data, for a different choice of the primordial density perturbation spectrum [15]. For example, as illustrated in Fig. 2 the CMB data can be fitted equally well by a flat world model with $\Omega_{\mathrm{m}}=1$ and $h \simeq 0.5$ if the primordial spectrum has a broken power-law form with $n \simeq 1$ for $k \lesssim 0.01 \mathrm{Mpc}^{-1}$, tilting to $n \simeq 0.9$ on smaller scales. Such a model would however predict the amplitude of matter fluctuations on the scale of $8 h^{-1} \mathrm{Mpc}$ to be $\sigma_{8} \simeq 1.1$, which is too high to match the observed abundances of galaxy clusters as well as measurements of weak gravitational lensing in a $\Omega_{\mathrm{CDM}}=1$ universe. However this is easily solved by noting that neutrinos are now known to be massive and thus naturally provide a small component of hot dark matter. The absolute mass scale is unknown but can be upto $1-2 \mathrm{eV}$ on the basis of both $\beta$-decay and $\beta \beta$ experiments [16. If for example the 3 types of neutrinos have a $(\sim$ common $)$ mass of 0.8 $\mathrm{eV}$ corresponding to $\Omega_{\nu}=0.12,{ }^{2}$ then as shown in Fig. 3 we can obtain an acceptable fit to LSS data with $\sigma_{8}=0.64$ which agrees with cluster abundances and weak lensing observations.

This model has $\Omega_{\mathrm{B}} h^{2}=0.021$ which is consistent with nucleosynthesis [2], Because the Hubble parameter is low (which ensures no 'age crisis'),

\footnotetext{
${ }^{2}$ The reason why other authors (e.g. ref.[11]) quote far more restrictive limits on neutrino masses on the basis of the same data is because they adopt 'priors' such as the higher HKP value of $h$ in their analyses [17].
}

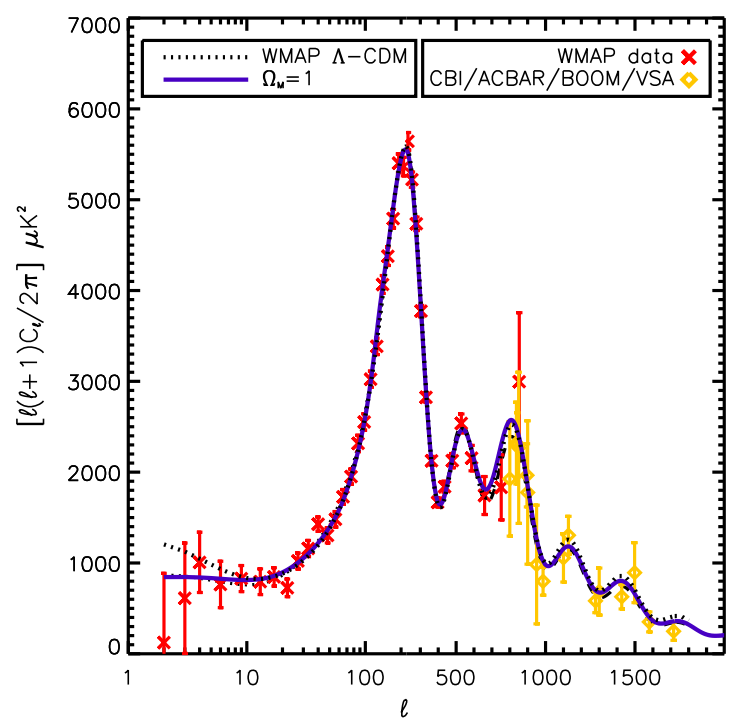

Figure 2. The CMB power spectrum for the bestfit $n \simeq 1 \Lambda \mathrm{CDM}$ model (dotted black line), and for a broken-power-law flat model with $\Omega_{\Lambda}=0$ (solid blue line), compared to data from WMAP and other experiments [15].

the baryon density is high enough to imply a cluster baryon fraction of $\Omega_{\mathrm{B}} / \Omega_{\mathrm{CDM}} \simeq 11 \%$, which is consistent with $\mathrm{X}$-ray observations of clusters [18. Although $h \simeq 0.5$ is not consistent with the HKP measurement $h=0.72 \pm 0.08$ based on the "cosmological distance ladder" 14, such a low value is in fact suggested by direct (and much deeper) determinations based on the SunyaevZeldovich effect in clusters, $h=0.54 \pm 0.03 \pm 0.18$ [19, and time delays in gravitationally lensed images of quasars, $h=0.48 \pm 0.03$ [20], although the systematic uncertainties here are large.

Clearly the observational situation is not as stable as would be desirable. In fact even the much touted 'concordance' between the different lines of evidence for the $\Lambda \mathrm{CDM}$ model is begining to unravel, e.g. analysis of the latest SNIa datasets 2122 clearly indicates a closed universe with $\Omega_{\mathrm{m}}+\Omega_{\Lambda}>1$, in conflict with the CMB data at $88-97 \%$ c.l. 23 . 

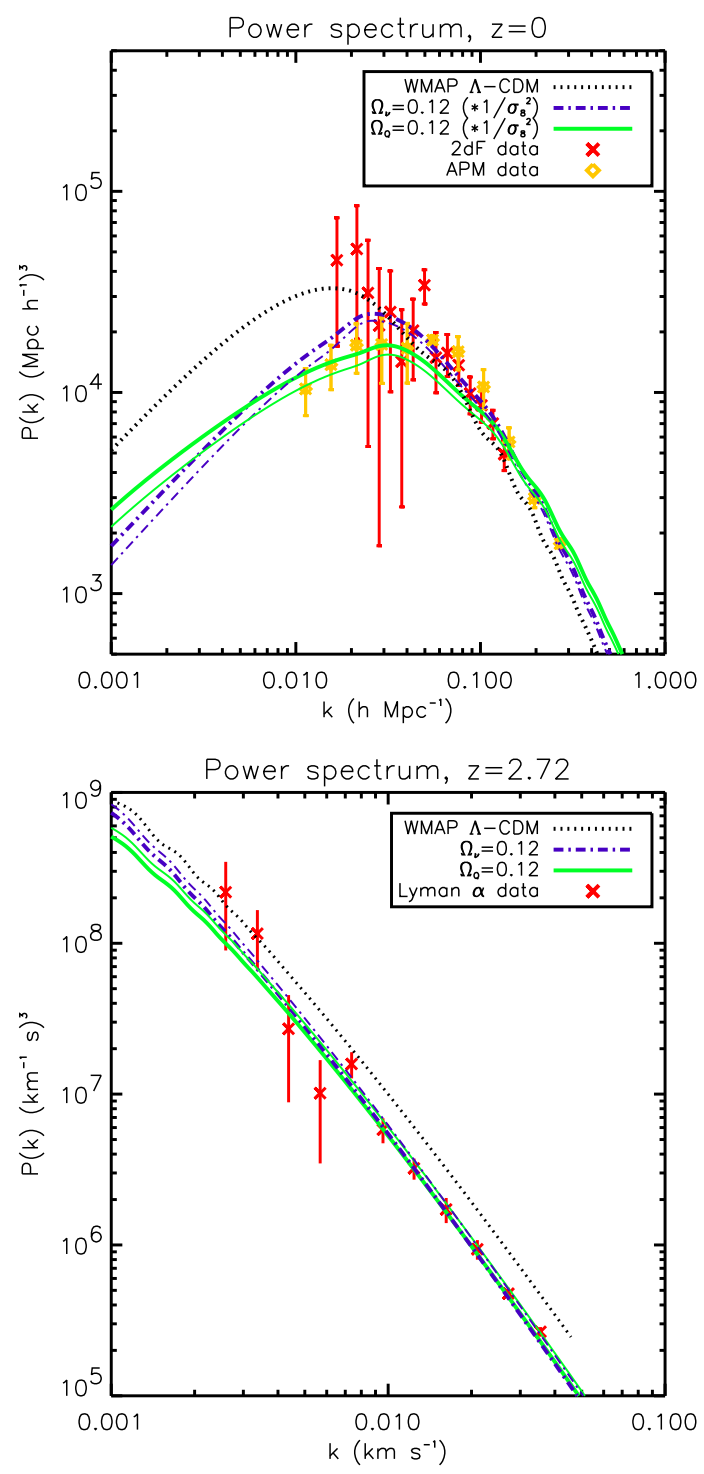

Figure 3 . The LSS power spectrum for the best-fit $n \simeq 1 \Lambda \mathrm{CDM}$ model (dotted black line), and for a broken power-law flat MDM model with $\Omega_{\Lambda}=0$ and $\Omega_{\nu}=0.12$ (dot-dashed blue line), compared to data from APM, $2 \mathrm{dF}$ and the Ly- $\alpha$ forest 15 . For the latter model a bias parameter of $1 / \sigma_{8} \simeq$ 1.6 has been adopted.

\section{Expectations for the primordial spec- trum from inflation}

It is often stated that the "simplest models of inflation" predict a (nearly) scale-invariant spectrum, thus justifying the adoption of such a spectrum in most analyses of CMB and LSS data. Indeed if inflation is driven by a single scalar field with a potential $V(\phi)$, then the generated scalar density perturbation has a power-law index [24]:

$n(k)=1-3 M^{2}\left(\frac{V^{\prime}}{V}\right)_{\star}^{2}+2 M^{2}\left(\frac{V^{\prime \prime}}{V}\right)_{\star}$

where $M \equiv\left(8 \pi G_{\mathrm{N}}\right)^{-1 / 2} \simeq 2.4 \times 10^{18} \mathrm{GeV}$ is the normalized Planck mass and $\star$ denotes that the derivatives wrt $\phi$ are to be evaluated when a mode of wavenumber $k$ crosses the 'Hubble radius' $H^{-1}$. Thus for a sufficiently 'flat potential' (as is necessary to achieve sufficient e-folds of inflation to solve the problems of the standard cosmology), the spectrum would be expected to have $n \simeq 1$.

Note however that the number of e-folds required to generate our present Hubble volume is

$N_{\star}(k) \simeq 51+\ln \left(\frac{k^{-1}}{3000 h^{-1} \mathrm{Mpc}}\right)$,

for phenomenologically acceptable choices of the inflationary scale and reheat temperature - the former is restricted to be $<2.8 \times 10^{16} \mathrm{GeV}$ [25] to respect the WMAP bound on tensor fluctuations, and the latter must be $<10^{9} \mathrm{GeV}$ in a supersymmetric theory in order not to overproduce gravitinos [26]. Thus fluctuations on the scales $(\sim 1-3000 \mathrm{Mpc})$ probed by LSS and CMB observations are generated just 40-50 e-folds before the end of inflation. It would be natural, especially in field-theoretical models of 'new inflation' where $\phi \ll M$, to expect the inflaton potential to begin to curve significantly as the end of inflation is approached. There are indeed attractive models of inflation in which the spectrum is significantly tilted in this region, e.g. in a model where the leading term in the potential is cubic [7], the spectral index is $n(k)=\left(N_{\star}-2\right) /\left(N_{\star}+2\right) \simeq 0.9$ at these scales $[8]$.

Moreover the anomalously small values of the low $\ell$ multipoles in the WMAP (and COBE) data suggest that the primordial density perturbation 
may have a cutoff on the scale of the present Hubble radius 272829313032, i.e. that inflation lasted just about long enough the produce a homogeneous patch as big as our present Hubble volume. There are also outliers or 'glitches' in the WMAP power spectrum suggestive of oscillatory features in the primordial density perturbation 3334. Both of these observations are in fact consistent with the idea of 'multiple inflation', which was a first attempt to take into account the effect of 'flat direction' scalar fields other than the inflaton on the inflationary density perturbation 35. It was noted that such fields are likely to undergo symmetry breaking during inflation and, by virtue of their gravitational coupling to the inflaton, to induce sudden changes in its mass. This will result in a step-like feature in the spectrum with associated oscillations, ${ }^{3}$ as was shown recently by solution of the governing Klein-Gordon equation 38 - see Fig 3 The consequences of such a feature in the primordial spectrum for parameter extraction from CMB and LSS data are presently under study [39].

It has been emphasized that the usual expectation for the nearly scale-invariant density perturbation from inflation is based on the assumption of an unchanging equation-of-state during the inflationary era [40. Given our ignorance of the actual physics of inflation, it would be overly simplistic to assume that the most trivial possibility was in fact realized in the very early universe. Moreover there are interesting anomalies in the data which seem unlikely to be all statistical flukes and which could well provide our first physical link to the process responsible for generating the primordial density perturbation. It is essential that these issues be studied further before we conclude that the 'standard model' of cosmology has indeed been established. What is very encouraging is that the expected increase in precision of forthcoming CMB experiments (especially at high $\ell$ s), together with measurements of polarisation, will be able to determine unambiguously if scale-invariance is indeed broken in the primordial spectrum [41].

\footnotetext{
$\overline{{ }^{3} \text { A similar feature arises }} 36$ if the inflaton evolves through a 'kink' - a discontinuity in its slope - although no physical model for this was given (see also ref. 37])
}

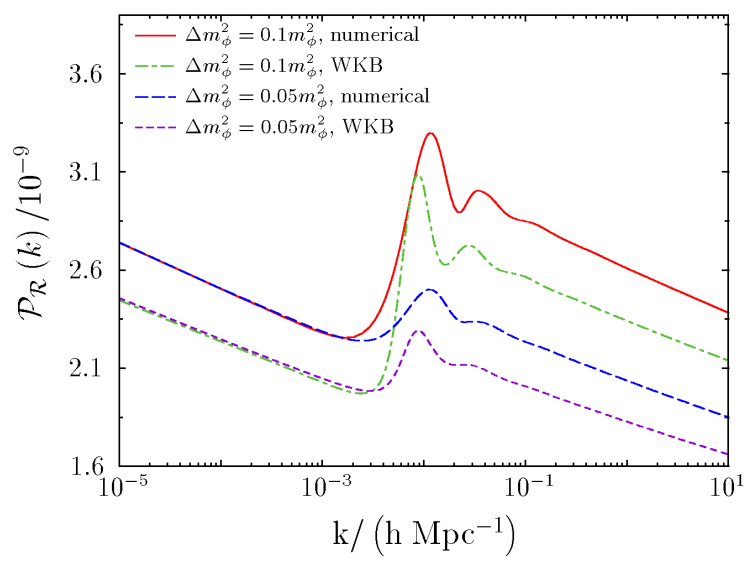

Figure 4. Comparison of the 1st-order WKB approximation with the numerically calculated exact spectrum of the density perturbation in the multiple inflation model, when the mass of the inflaton undergoes a sudden change of 5-10\% 38.

\section{Acknowledgements}

I wish to thank all my collaborators in the work reported on here, and the organisers of this stimulating meeting for the invitation to present a somewhat unorthodox view.

\section{REFERENCES}

1. see e.g., T. Padmanabhan, 'Structure Formation in the Universe' (CUP, 1993)

2. B. Fields and S. Sarkar, in 'Review of Particle Physics', S. Eidelman et al. (Particle Data Group), Phys. Lett. B592 (2004) 1 arXiv:astro-ph/0406663.

3. S.D.M. White, G. Efstathiou and C.S. Frenk, Mon. Not. Roy. Astron. Soc. 262 (1993) 1023.

4. D. Scott, J. Silk and M. J. White, Science 268 (1995) 829 arXiv:astro-ph/9505015.

5. N.A. Bahcall, J.P. Ostriker, S. Perlmutter and P.J. Steinhardt, Science 284 (1999) 1481 arXiv:astro-ph/9906463.

6. see e.g. P.J.E. Peebles and B. Ratra, Rev. Mod. Phys. 75 (2003) 559 arXiv:astro-ph/0207347. 
7. G.G. Ross and S. Sarkar, Nucl. Phys. B 461 (1996) 597 arXiv:hep-ph/9506283

8. J.A. Adams, G.G. Ross and S. Sarkar, Phys. Lett. B $391 \quad$ (1997) 271 arXiv:hep-ph/9608336.

9. G. Jungman, M. Kamionkowski, A. Kosowsky and D.N. Spergel, Phys. Rev. D 54 (1996) 1332 arXiv:astro-ph/9512139.

10. G. Efstathiou and J.R. Bond, Mon. Not. Roy. Astron. Soc. 304 (1998) 75 arXiv:astro-ph/9807103.

11. D.N. Spergel et al. [WMAP collab], Astrophys. J. Suppl. $148 \quad$ (2003) 175 arXiv:astro-ph/0302209.

12. W.J. Percival et al. [2dFGRS collab.], Mon. Not. Roy. Astron. Soc. 327 (2001) 1297 arXiv:astro-ph/0105252.

13. S. Perlmutter et al. [Supernova Cosmology Project collab.], Astrophys. J. 517 (1999) 565 arXiv:astro-ph/9812133.

14. W. L. Freedman et al. [Hubble Key Project collab.], Astrophys. J. 553 (2001) 47 arXiv:astro-ph/0012376.

15. A. Blanchard, M. Douspis, M. RowanRobinson and S. Sarkar, Astron. Astrophys. 412 (2003) 35 arXiv:astro-ph/0304237.

16. F. Feruglio, A. Strumia and F. Vissani, Nucl. Phys. B 637 (2002) 345 (Addendum-ibid. B 659 (2003) 359) arXiv:hep-ph/0201291.

17. O. Elgaroy and O. Lahav, JCAP 0304 (2003) 004 arXiv:astro-ph/0303089.

18. R. Sadat and A. Blanchard, As$\begin{array}{lllll}\text { tron. Astrophys. } & \mathbf{3 7 1} & \text { (2001) } & 19\end{array}$ arXiv:astro-ph/0102010.

19. E.D. Reese, in 'Measuring and Modeling the Universe', ed. W. Freedman (CUP, 2004) p.138 arXiv:astro-ph/0306073.

20. C.S. Kochanek and P.L. Schechter, in 'Measuring and Modeling the Universe', ed. W. Freedman (CUP, 2004) p.117 arXiv:astro-ph/0306040.

21. J.L. Tonry et al. [Supernova Search Team collab.], Astrophys. J. 594 (2003) 1 arXiv:astro-ph/0305008.

22. A.G. Riess et al. [Supernova Search Team collab.], Astrophys. J. 607 (2004) 665 arXiv:astro-ph/0402512.

23. T.R. Choudhury and T. Padmanab- han, Astron. Astrophys. 429 (2005) 807 arXiv:astro-ph/0311622.

24. A.R. Liddle and D.H. Lyth, 'Cosmological Inflation and Large-scale Structure' (CUP, 2000).

25. V. Barger, H. S. Lee and D. Marfatia, Phys. Lett. B 565 (2003) 33 arXiv:hep-ph/0302150.

26. J. R. Ellis, D. V. Nanopoulos and S. Sarkar, Nucl. Phys. B 259 (1985) 175.

27. S.L. Bridle, A.M. Lewis, J. Weller and G. Efstathiou, Mon. Not. Roy. Astron. Soc. 342 (2003) L72 arXiv:astro-ph/0302306.

28. P. Mukherjee and Y. Wang, Astrophys. J. 599 (2003) 1 arXiv:astro-ph/0303211.

29. C.R. Contaldi, M. Peloso, L. Kofman and A. Linde, JCAP 0307 (2003) 002 arXiv:astro-ph/0303636.

30. J.M. Cline, P. Crotty and J. Lesgourgues, JCAP $0309 \quad$ (2003) 010 arXiv:astro-ph/0304558.

31. B. Feng and X. Zhang, Phys. Lett. B 570 (2003) 145 arXiv:astro-ph/0305020.

32. A. Shafieloo and T. Souradeep, Phys. Rev. D 70 (2004) 043523 arXiv:astro-ph/0312174.

33. H.V. Peiris et al. [WMAP collab.], Astrophys. J. Suppl. $148 \quad$ (2003) 213 arXiv:astro-ph/0302225.

34. D. Tocchini-Valentini, M. Douspis and J. Silk, arXiv:astro-ph/0402583

35. J.A. Adams, G.G. Ross and S. Sarkar, Nucl. Phys. B $\mathbf{5 0 3}$ (1997) 405 arXiv:hep-ph/9704286.

36. A.A. Starobinsky, JETP Lett. 55 (1992) 489. [Pisma Zh. Eksp. Teor. Fiz. 55 (1992) 477].

37. J.A. Adams, B. Cresswell and R. Easther, Phys. Rev. D 64 (2001) 123514 arXiv:astro-ph/0102236.

38. P. Hunt and S. Sarkar, Phys. Rev. D 70 (2004) 103518 arXiv:astro-ph/0408138.

39. M. Douspis, J. Dunkley, P. Hunt, A. Morgan and S. Sarkar, in preparation.

40. L.M. Wang, V.F. Mukhanov and P.J. Steinhardt, Phys. Lett. B $414 \quad$ (1997) 18 arXiv:astro-ph/9709032.

41. J. R. Bond, C. R. Contaldi, A. M. Lewis and D. Pogosyan, Int. J. Theor. Phys. 43 (2004) 599 arXiv:astro-ph/0406195. 